Proc. of the 11 Int. School on Theoretical Physics Symmetry and Structural Properties of Condensed Matter, Rzeszów 2014

\title{
An Investigation of the The dynamic Young Moduli of Twisted Fibres
}

\author{
A. WASILEWSKI* AND T. WięCEK \\ Rzeszów University of Technology, al. Powstanców Warszawy 12, 35-329 Rzeszów, Poland
}

\begin{abstract}
This paper describes a modified laser system to measure the dynamic Young modulus and logarithmic decrement of damping for twisted fibers. A modified impulse mechanical spectrometer was designed and built by the authors. The effect of twisting on the values of the dynamic Young modulus and logarithmic decrement of damping was studied. The dynamic Young modulus and logarithmic decrement of damping were investigated for long PET fibers with a $200 \mu \mathrm{m}$ diameter. This experimental method does not require calibration because it contains standard length which is the wavelength of the laser beam.
\end{abstract}

DOI: 10.12693 /APhysPolA.128.231

PACS: 62.20.de, 62.20.D-

\section{Introduction}

Composite materials are widely used in industry. Designing composite materials requires knowledge of the mechanical properties of single fibers. The dynamic Young modulus and logarithmic decrement of damping are just two of many mechanical parameters of the fiber $[1,2]$ and present the determination of the dynamic Young modulus for a non-twisted fiber using an impulse mechanical spectrometer (IMS). One-dimensional mechanical components are often subjected to tension and torsion. Theoretical results published in paper [3] were used to obtain analytical expressions for the maximum contact stresses induced in cables subjected to tension and twisting. On a small scale, the response of hollow and filled carbon nanotubes subjected to tension and twisting is investigated using classical molecular dynamic simulations [4]. Paper [5] presents the plastic deformation of pure copper under tension and twisting conditions. A fiber composite may be twisted or non-twisted. To this end, research was conducted into how the dynamic Young modulus and logarithmic decrement of damping change for a twisted fiber,

\section{The dynamic Young modulus}

The dynamic modulus is the ratio of stress to strain under vibratory conditions. In purely elastic materials stress and strain occur in phase, so that the response of one occurs simultaneously with the other. In purely viscous materials there is a phase difference between stress and strain, with strain lagging stress by $90^{\circ}(\pi / 2$ radians). This is known as phase lag. The behavior of viscoelastic materials lies between that of purely viscous and purely elastic materials, and exhibits some phase lag. Stress and strain in a viscoelastic material can be represented using the following expressions $[6,7]$ :

${ }^{*}$ corresponding author; e-mail: optlabwa@prz.edu.pl

$$
\begin{aligned}
& \text { stress: } \quad \sigma=\sigma_{0} \sin (\omega t), \\
& \text { strain: } \varepsilon=\varepsilon_{0} \sin (\omega t-\varphi),
\end{aligned}
$$

where $\omega$ is the frequency of strain oscillation, $t$ is the time, $\varphi$ is phase lag between stress and strain.

In viscoelastic solids the tensile storage modulus measures the stored energy (elastic component) while the loss modulus measures the energy dissipated as heat (viscous component). The tensile storage and loss moduli are defined as follows:

$$
\begin{aligned}
& \text { storage: } E^{\prime}=\frac{\sigma_{0}}{\varepsilon_{0}} \cos (\varphi), \\
& \text { loss: } E^{\prime \prime}=\frac{\sigma_{0}}{\varepsilon_{0}} \sin (\varphi),
\end{aligned}
$$

The modulus $E^{*}$ can be expressed using complex notation as follows:

$$
E^{*}=E^{\prime}+\mathrm{i} E^{\prime \prime}
$$

where $\mathrm{i}$ is the imaginary unit.

\section{Experimental setup}

A method to determine the dynamic elastic modulus for a single twisted fiber is presented below. This is a modified impulse mechanical spectrometer $[1,2]$. The method, based on a laser diffractographic technique [811], provides dynamic elastic modulus measurements. The apparatus, specially designed to measure the dynamic elastic modulus for a fiber (Fig. 1), consists of a mechanical setup and a diffraction measurement system using a Ne-He laser and detector. The fiber sample is fixed in a holder (1) at one end and a weight is attached to the other through a series of elements $(2,4$ and $5)$. The dynamic elastic modulus is determined using the fiber longitudinal vibration of elastic deformation. The fiber under test, loaded with an additional weight (elements $2,4,5)$, can be made to oscillate by subjecting it to an impulse force such as an impact to the frame (2) from a falling steel ball. The initial load applied to the fiber was not greater than $0.5 \mathrm{cN} /$ tex. As the bearing movable rod (4) vibrates it changes the width of the slit while the laser beam passing through the slit generates a diffraction pattern. The setup for dropping the metal 
ball consists of an electromagnet and a glass tube (10). A metal ball is placed at the top of the glass tube and held in place by the electromagnet when the current is switched on. When the current is switched off the ball (9) travels along the tube and drops onto the connecting frame (2) in such a way so as not to interfere with the laser beam (8).

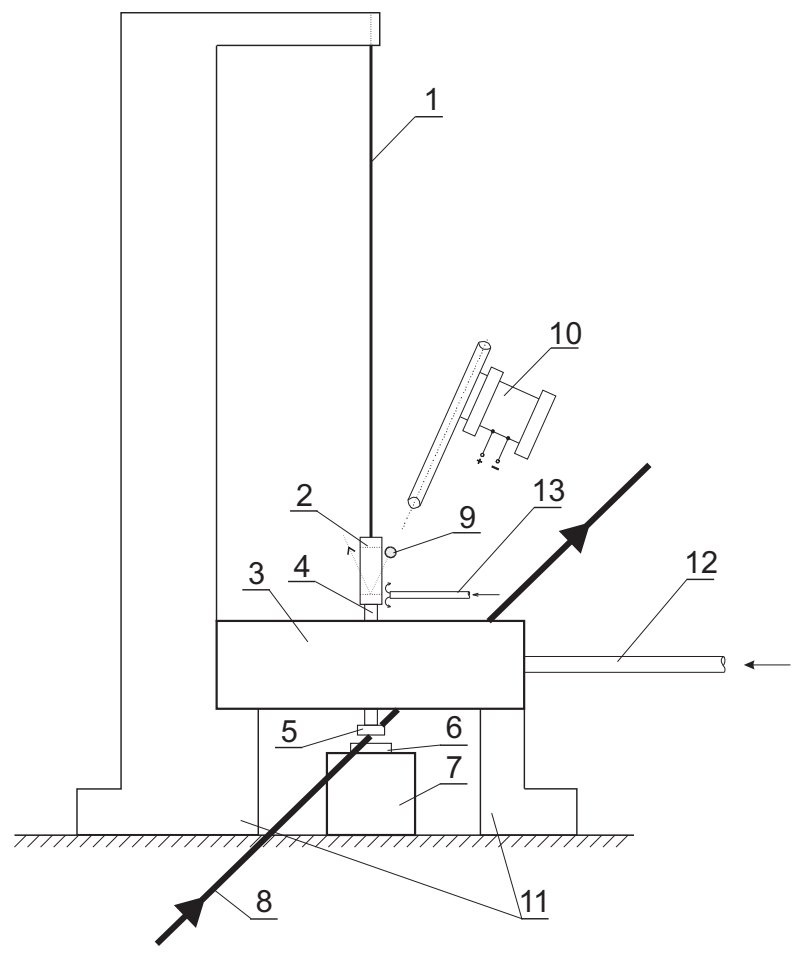

Fig. 1. Schematic of the impulse mechanical spectrometer: 1 - fiber, 2 - connecting frame, 3 - air bearing, 4 - bearing movable rod, 5 - movable edges, 6 fixed edges, 7 - support table with adjustable position, 8 - laser beam, 9 - small metal ball, 10 - set up for dropping the metal ball, 11 - holder, 12 - compressed air inlet, 13 - anti-rotation airflow.

An experimental technique can be used to analyze a fiber longitudinal vibrations. In order to investigate this purely longitudinal vibration, any transverse vibrations in the mechanical spectrometer must be effectively eliminated. For this reason the impulse mechanical spectrometer is constructed with an air and frictionless bearing, which only allows the fiber to vibrate vertically. The air bearing (9) should be frictionless in the desired direction. This is possible when an air bag is placed between the moving surfaces. During the experiment the slit affects the diffraction image, specifically the light intensity in the zero fringe, where detector $\mathrm{D}$ converts the optical signal into an electrical one. This is then amplified, digitized using a 12-bit A/D converter, and processed using standard off-the-shelf software (Fig. 2).

The time-domain spectrum is analyzed using mathematical functions of the form $g(t)[12]$ with parameters: $A, \beta, \omega, \varphi$, a)

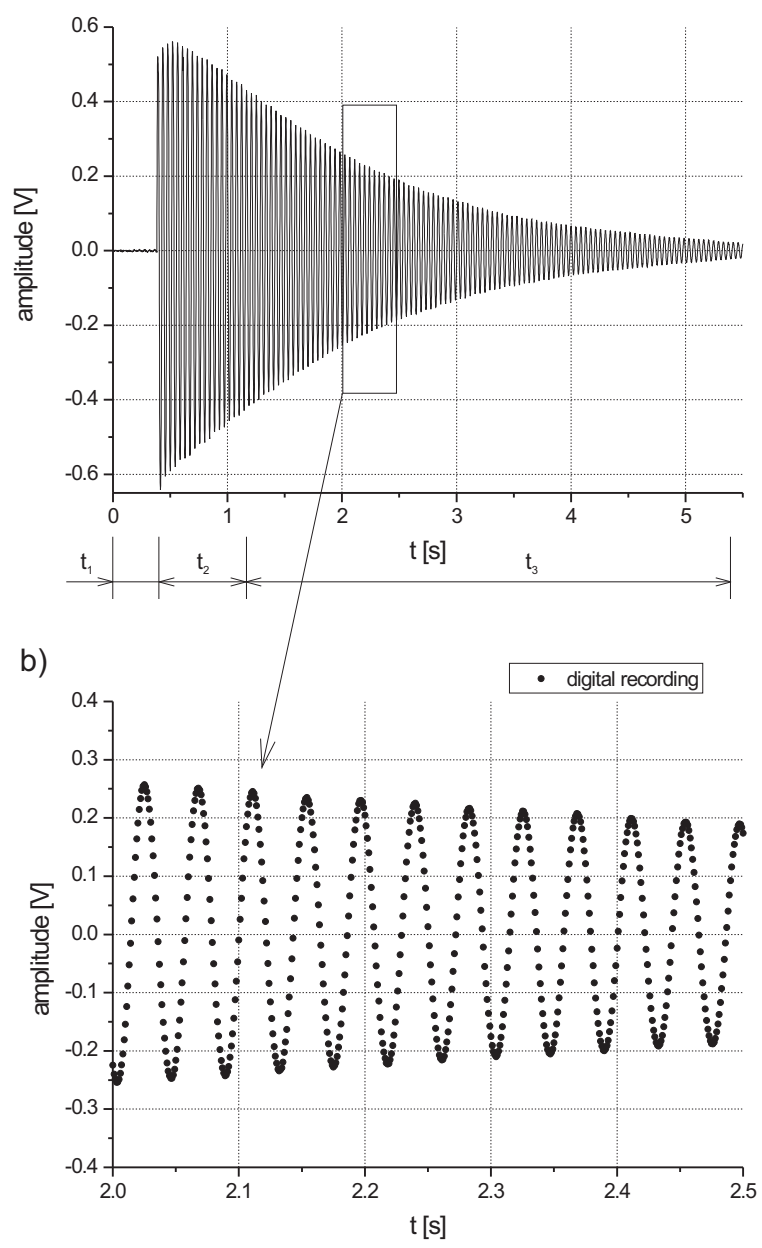

Fig. 2. Amplitude of vibration versus time, a) total spectrum, b) expanded view of a selected portion of trace.

$$
g(t)=A \mathrm{e}^{-\beta t} \sin (\omega t+\varphi),
$$

where $A$ is the amplitude, $\beta$ is the damping coefficient, $\omega$ is the resonant frequency, $\varphi$ is the phase, $t$ is time. Initial values for the parameters were determined graphically. By placing dozens of periods of mechanical loss spectra and a curve-fitting function on one chart we can adjust parameters $(A, \beta, \omega, \varphi)$, until the two traces are superimposed. By providing initial values for the parameters the software can generate the exact ones. The quality of this fit was analyzed graphically by comparing on one graph the mechanical loss spectra. In addition, the least squares method was also used for verification. The resultant parameters $\beta, \omega$, allow the Young modulus $E$, and dimensionless logarithmic decrement of damping $(\delta)$ to be calculated. In the calculation the mass of the fiber is neglected because it is insignificant compared to the mass of the load.

$$
\sigma=\frac{F}{S} \quad \varepsilon=\frac{\Delta L}{L} \quad k=\frac{F}{\Delta L},
$$




$$
\omega_{0}^{2}=\frac{k}{m} \quad \omega^{2}=\omega_{0}^{2}-\beta^{2},
$$

where $\sigma$ is stress, $F$ is force, $S$ is cross-sectional area of the fiber $\left(S=\pi d_{n}^{2} / 4\right)$, $\varepsilon$ is relative elongation, $L$ is length of the sample, $k$ is coefficient of elasticity, $m$ is mass of the load. The dynamic Young modulus $E_{\text {IMS }}$ and logarithmic decrement of damping $\delta$ were calculated from the following dependences [13]:

$$
\begin{aligned}
& E_{\mathrm{IMS}}=\frac{\sigma}{\varepsilon}=\frac{F \cdot L}{S \cdot \Delta L}=m \frac{4 L}{\pi d_{n}^{2}}\left(\omega^{2}+\beta^{2}\right), \\
& \delta=\frac{2 \pi \beta}{\omega} .
\end{aligned}
$$

\section{Results}

A PET fiber was mounted using an adhesive. Its length was $168 \mathrm{~mm}$ and it had a diameter of $200 \mu \mathrm{m}$. The fiber thickness was specified by the manufacturer (Rhodia). The fiber was loaded with a static mass of $92.95 \mathrm{~g}$. A metal ball of mass $0.9 \mathrm{~g}$ falling from a distance of $10 \mathrm{~cm}$ was used to initiate the vibrations. The time dependent signal from the photodetector is shown in Fig. 2. The vibration starts at time $t_{1}$, when the falling ball hits the frame. The initial part of the response, from $t_{1}$ to $t_{2}$ is ignored because the amplitude is too great to be transferred linearly by the electronics. A damped harmonic motion can be clearly observed which is in agreement with Eq. (6). A set of parameters $(A, \beta, \omega, \varphi)$ was obtained using software from Microcal Origin. The software calculated the angular frequency of vibration as $\omega=146.33 \mathrm{rad} \mathrm{s}^{-1}$ and the decay rate as $\beta=0.660 \mathrm{~s}^{-1}$ for a non-twisted fiber. The dynamic Young modulus was estimated as $1.064 \times 10^{10} \mathrm{~Pa}$ using Eq. (9).

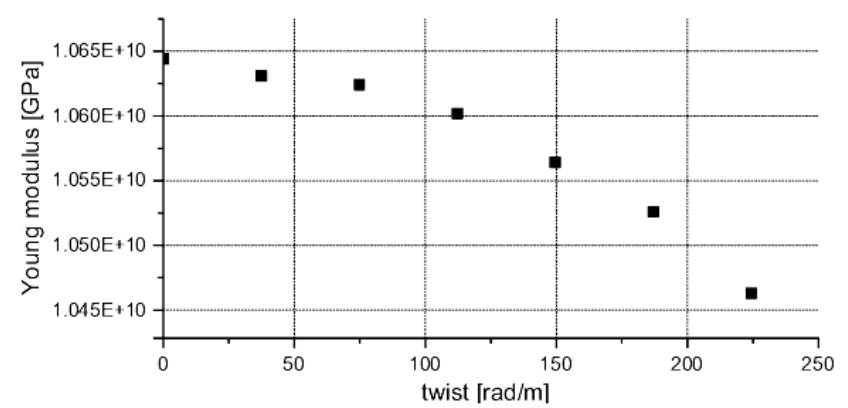

Fig. 3. The dynamic Young modulus versus twisted fibers..

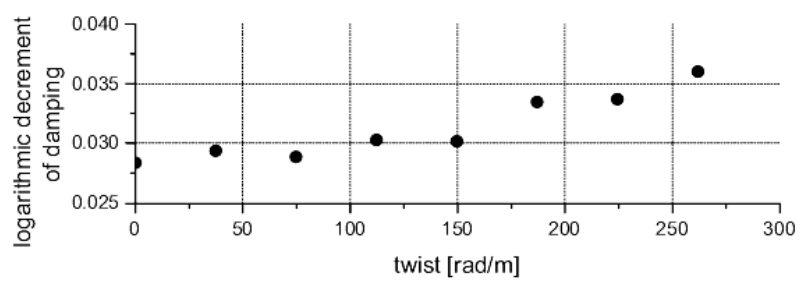

Fig. 4. Logarithmic decrement of damping versus twisted fibers.

\section{Conclusions}

On the basis of the obtained results we can conclude the following:

1. Using our laser system, we can determine the Young modulus without the need for calibration. This is because the wavelength used in our mathematical formulae is a standard measurement unit of length.

2. A parameter set consisting of the static and the dynamic Young moduli for longitudinal vibrations has an application in the materials engineering industry.

3. The extension of the fiber is in the elastic region.

4. The non-contact measurement of the slit width is performed using a diffractometer.

5. The Young modulus decreases with increasing fiber twist.

\section{References}

[1] T. Więcek, Composites Part A 60, 1 (2014).

[2] A. Wasilewski, T. Więcek, L.B. Magalas, Solid State Phenom. 89, 343 (2003).

[3] K. Kumar, J. Botsis, Trans. ASME 68, 432 (2001).

[4] B.-W. Jeong, J.-K. Lim, S.B. Sinnott, Appl. Phys. Lett. 91, 093102 (2007).

[5] C. Wang, F. Li, L. Wei, Y. Yang, J. Dong, Mater. Sci. Eng. A 571, 95 (2013).

[6] I.M. Ward, Mechanical Properties of Solid Polymers, Wiley, New York 1971.

[7] A. Demšar, V. Bukošek, A. Kljun, Fibres Textiles East. Eur. 18, 29 (2010).

[8] M. Bass, Handbook of Optics, McGraw-Hill, New York 1995

[9] J.R. Meyer-Arendt, Introduction to Classical and Modern Optics, Prentice-Hall, New Jersey 1972.

[10] W.T. Cathey, Optical Information Processing and Holography, Wiley, New York 1974.

[11] D. Malacara, B.J. Thompson, Handbook of Optical Engineering, Marcel Dekker, New York 2001.

[12] A.K. Jonsher, Universal Relaxation Law, Chelsea Dielectrics Press, London 1976.

[13] C.W. De Silva, Vibration. Fundamentals and Practice, CRC Press, Boca Raton (FL) 2000. 